\title{
ADMINISTRACIÓN COLABORATIVA Y EN RED
}

\section{Jordi Graells-Costa}

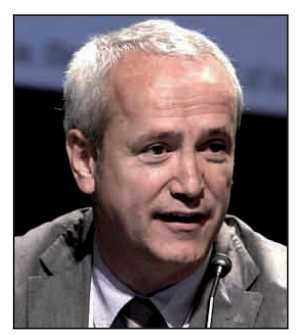

Jordi Graells-Costa, experto en comunicación web, redes sociales y gestión del conocimiento, es responsable de contenidos e innovación en la Generalitat de Catalunya (Gencat). Premio Nadal Batle de la Universidad de Illes Balears, sobre TIC, en su cuarta edición de 2002. Ha publicado y participado en las publicaciones: Innovar $x$ internet (EAPC, 2004), El trabajo colaborativo en la Administración (Departamento de Justicia, Gencat. Barcelona, febrero de 2010) y Guía de usos y estilo en las redes sociales de la Gencat (Barcelona, junio de 2010).

http://graells.cat

\section{Resumen}

Las pesadas estructuras de las administraciones públicas se ven obligadas a evolucionar ante la omnipresencia de las redes sociales en todos los ámbitos de la sociedad. Las administraciones deben perder su desconfianza hacia la web 2.0 y asumir los cambios que representan nuevas vías de comunicación con los ciudadanos y entre los propios funcionarios. Las redes sociales fomentan participación, transparencia e innovación a todos los niveles.

\section{Palabras clave}

Redes sociales, Web 2.0, Administración pública, Cambio, Colaboración, Communication, Gobierno digital, Oportunidades, Riesgos, Ventajas.

\section{Title: Collaborative public administration on the network}

\section{Abstract}

The weighty structures of government are forced to evolve due to the ubiquity of social networks in all areas of society. Governments should lose their distrust of web 2.0 and assume the changes brought about by these new ways of communicating with the public and among officials. Social networks favour participation, transparency and innovation at all levels.

\section{Keywords}

Social networking, Web 2.0, Public administration, Change, Collaboration, Communication, Digital government, Opportunities, Risks, Benefits.

Graells-Costa, Jordi. “Administración colaborativa y en red". El profesional de la información, 2011, mayo-junio, v. 20, n. 3, pp. 345-347.

Las redes sociales son algo más que unos meros canales de comunicación. A estas alturas empiezan a ser canales de atención ciudadana, ya que permiten intercambiar conocimiento además de contenidos. Los ciudadanos usuarios de las redes sociales crean comunidades y participan de la elaboración, distribución y valoración crítica de los servicios y de las políticas públicas y no se limitan sólo a usarlos. Valga como ejemplo la reciente aparición del canal de Twitter 012 de la Generalitat de Catalunya http://twitter.com/012

La Administración mira con sorpresa, con falta de conocimiento o incluso desconfianza las redes sociales. La sola presencia de algunos de sus organismos en Facebook, Twitter, YouTube... nos ha hecho recordar el estado de choque emocional similar -y sólo un poco- a la época cuando llegó internet y había que decidir qué empleados públicos tendrían acceso y para qué. Las redes generan nuevos espacios públicos de comunicación y debate que alteran las jerarquías convencionales.

Las redes sociales generan nuevos espacios públicos de comunicación y debate que alteran las jerarquías convencionales

Y es que, tradicionalmente, la comunicación y la relación le cuestan a la Administración. Imaginémonos pues ahora, que lo deba hacer encima en entornos externos promovidos por otros, como Facebook o Twitter, fuera de sus procedimientos rígidos de control. 


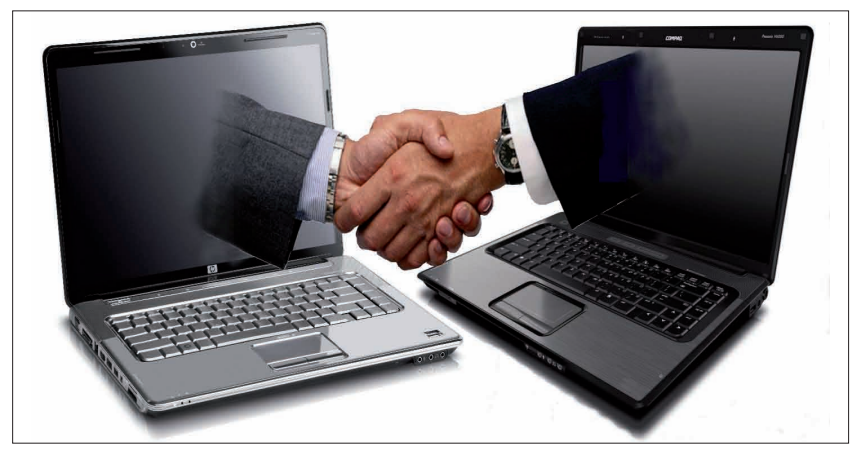

Estas escapadas de la Administración en internet han dado lugar inicialmente a unos contenidos flojos porque no respondían a objetivos estratégicos fijados previamente para definir los hitos a conseguir en estos espacios de relación.

Nos encontramos además con que la Administración no entra dentro de los planes de negocio de las redes sociales. La información y el conocimiento que puede verter en ellas no encajan, de entrada, con los contenidos o la publicidad que sirven para financiar las redes. Por lo tanto, las empresas que las promueven no conciben la Administración como un cliente a quien haya que mimar especialmente.

Precisamente estos objetivos más sociales hacen que se deba fomentar un entorno riguroso y profesional de actuación específica del sector público en las redes sociales, preservando su carácter abierto y garantizando la seguridad y privacidad de todos los intervinientes. La publicación de la Guía de usos y estilo en las redes sociales de la Generalitat de Catalunya cumple esta función, porque establece las pautas para homogeneizar la presencia de la Generalitat en las redes mediante un sistema profesional y transferible que tiene en cuenta la protección de datos sensibles.

Se trata de que las administraciones vean las redes sociales no como unas plataformas extrañas, ni como un medio ideal para conseguir un crecimiento rápido de la audiencia, sino como unas herramientas aliadas. Son instrumentos muy aptos para compartir, participar, escuchar y conversar. El reto es que la Administración use esos medios que se están convirtiendo en el espacio natural para relacionarse con la ciudadanía y otros organismos que aportan valor público.

La web social nos ayudará a conformar nuevos espacios para la Administración. De puertas adentro, pueden ser muy efi-

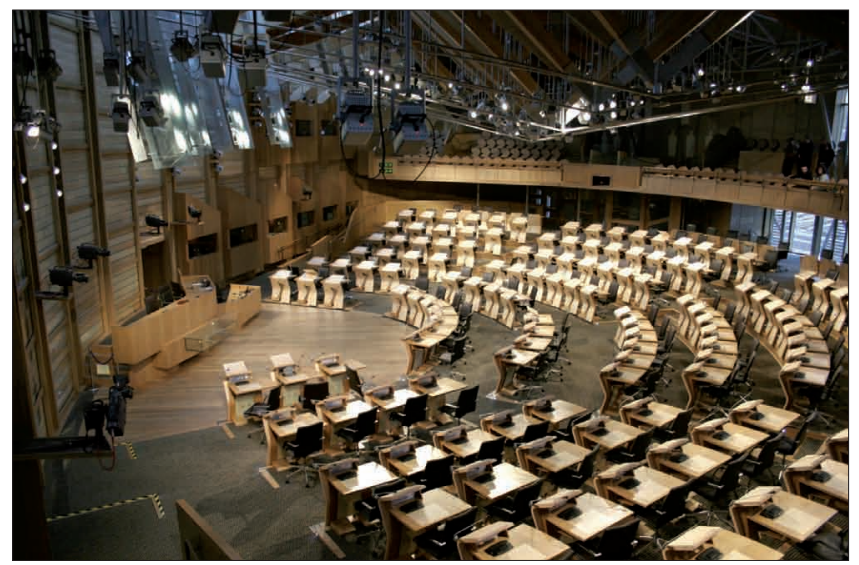

Moderna sala de debates del Parlamento de Escocia (2004), obra del arquitecto español Enric Miralles caces para promover estrategias de colaboración entre los trabajadores públicos para mejorar servicios y la organización misma. Externamente, pueden definir un nuevo espacio público donde las relaciones entre los diversos agentes, que están todavía para construir, podrán innovar el entorno social y profesional. Por eso, es fácil imaginar que el escenario cambiará radicalmente.

Al igual que sucede en los otros canales de atención ciudadana, en las redes sociales las diferentes administraciones deben relacionarse con su público destinatario. La Administración local lo atenderá en aquellos servicios de proximidad que provee habitualmente, y la autonómica en aquéllos otros que son objeto de su competencia en el ámbito territorial de la Comunidad.

A modo de conclusión, podemos decir que las redes sociales están transformando las organizaciones, en general, y las administraciones, en particular, modificando tanto la relación con los mercados y los ciudadanos como la gestión de su conocimiento interno, tan importante para la innovación de servicios.

Las redes sociales nos ayudarán a conformar nuevos espacios para la Administración

\section{Bibliografía}

Blaser, Britt; Weinberger, David; Trippi, Joe. "Digital government through social networks: how citizens can aggregate their money and votes to define digital government" http://portal.acm.org/citation.cfm?id=1556185

Blog Gencat. Predicciones para el 2011 sobre Administración y redes sociales. Opiniones de Cristina Aced, Ana Cabañas, Simon Collister, Marc Cortés, Roc Fages, José-Antonio Del-Moral, Tona Pou, Dolors Reig, Genís Roca, María Salido, Isabel Troytiño y Marc Vidal.

Generalitat de Catalunya, DG d’Atenció Ciutadana i Difusió. http://blocs.gencat.cat/.../predicciones-para-el-2011-sobreadministracion-y-redes-sociales.pdf

Bodin, Örjan; Crona, Beatrice I. "The role of social networks in natural resource governance: What relational patterns make a difference?". Global environmental change, 2009, v. 19, n. 3, August, pp. 366-374. DOI:10.1016/ j.gloenvcha.2009.05.002

http://myweb.fsu.edu/jastallins/courses/research/bodin. $p d f$

Chamorro-Marín, Rafael. "Oportunidades, riesgos y ventajas. Las redes sociales y las administraciones públicas" [presentación]. En: Tecnimap, 6-9 abril 2010.

http://www.slideshare.net/rchamorro/las-redes-sociales-ylas-administraciones-pblicas-3564337

Fernández, Laura. 2011, ¿el año de las Administraciones Públicas en las redes sociales?, 29 diciembre 2010.

http://www.euskadinnova.net/es/innovacion-social/noti cias/2011-administraciones-publicas-redes-sociales/7255. aspx 
Freire, Juan. "Redes sociales: ¿̇modelos organizativos o servicios digitales?". El profesional de la información, 2008, v. 17, n. 6, noviembre-diciembre, pp. 585-588.

DOI: 10.3145/epi.2008.nov.01

http://www.elprofesionaldelainformacion.com

Generalitat de Catalunya. Guia d'usos i estil a les xarxes socials de la Generalitat de Catalunya, 3a ed. Barcelona, abril de 2011.

http://www.gencat.cat/web/meugencat/cas/xarxes.htm

Moynihan, Donald P.; Pandey, Sanjay K. "The ties that bind: social networks, person-organization value fit, and turnover intention". Journal of public administration research and theory, v. 18, n. 2, pp. 205-227.

http://jpart.oxfordjournals.org/content/18/2/205.full. pdf+html

Ortiz-Sánchez, Iñaki. Las redes sociales en la Administración pública, 18 abril 2010.

http://eadminblog.net/post/2010/04/18/las-redes-socia les-en-la-administracion-publica
Sapena, Sisco. Las administraciones públicas deben convertirse en redes sociales, 7 abril 2011.

http://www.rrhhdigital.com/ampliada. . $h p$ ?sec $=45 \& i d=$ 76144

Tecnimap. Las redes sociales son herramientas que cambiarán las relaciones entre ciudadanos y Administración. Reseñas, presentaciones y audios de Tecnimap 2010, Zaragoza, 6-9 de abril 2010.

http://www.tecnimap.es/node/19165

Tesoro, José-Luis. "Entrevista a Dolors Reig: e-Gobierno y participación de las administraciones públicas en redes sociales". Boletín de la Organización de los Estados Americanos, n. 57, septiembre 2010.

http://www.dreig.eu/caparazon/2010/09/08/e-gobiernoredes-sociale/
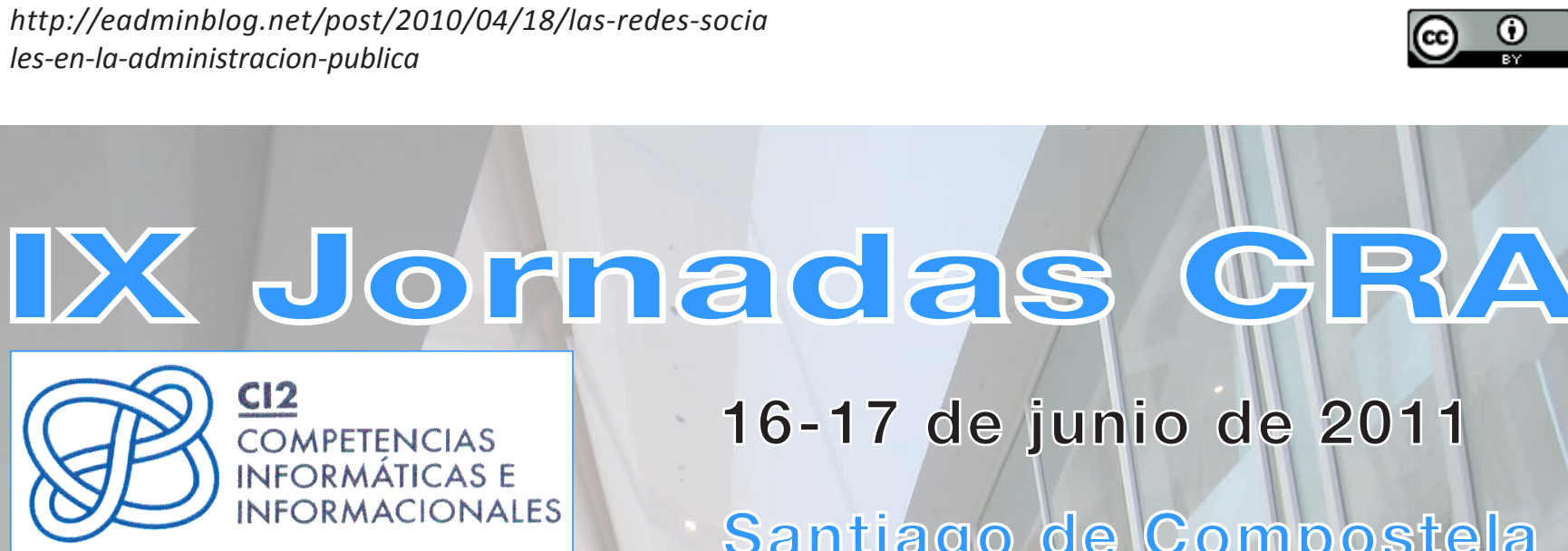

\section{$\mathrm{Cl} 2$}

COMPETENCIAS

INFORMÁTICAS E

INFORMACIONALES
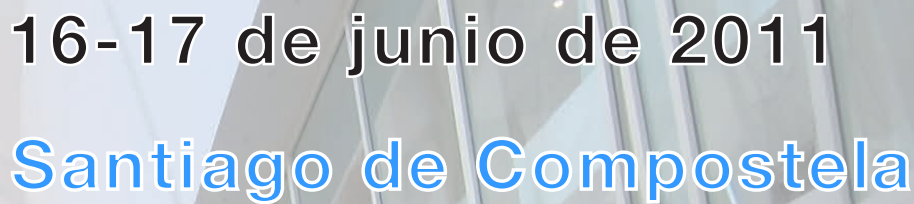

Por cuarto año consecutivo, dos comisiones sectoriales de la Conferencia de Rectores de las Universidades Españolas (Crue),

- Red de Bibliotecas Universitarias Españolas (Rebiun), y

- Tecnologías de la información y las Comunicaciones (TIC)

organizan conjuntamente las IX Jornadas CRAI, dedicadas a difundir el concepto de Centro de Recursos para el Aprendizaje y la Investigación.

Acogidas por la Universidade de Santiago de Compostela estarán dedicadas a la:

Evaluación y acreditación de las competencias informáticas e informacionales

Las jornadas se dirigen a toda la comunidad universitaria pero de manera especial todos aquellos que desarrollan su labor en el ámbito de los servicios de apoyo al aprendizaje, la docencia y la investigación.

\section{Ven a Santiago y aprovecha para visitar la espectacular recién estrenada Cidade da Cultura}

